\title{
Deblurring of noisy iris images in iris recognition
}

\author{
Shahrizan Jamaludin', Nasharuddin Zainal ${ }^{2}$, W. Mimi Diyana W. Zaki ${ }^{3}$ \\ ${ }^{1}$ Department of Maritime Technology, Faculty of Ocean Engineering Technology and Informatics, \\ Universiti Malaysia Terengganu, Malaysia \\ ${ }^{2,3}$ Department of Electrical, Electronic and Systems Engineering, Faculty of Engineering and Built Environment, \\ Universiti Kebangsaan Malaysia, Malaysia
}

\section{Article Info}

Article history:

Received Feb 1, 2020

Revised Apr 8, 2020

Accepted May 15, 2020

\section{Keywords:}

Blurry image

Deblurring

Iris pattern

Iris recognition

Wiener filter

\begin{abstract}
Iris recognition used the iris features to verify and identify the identity of human. The iris has many advantages such as stability over time, easy to use and high recognition accuracy. However, the poor quality of iris images can degrade the recognition accuracy of iris recognition system. The recognition accuracy of this system is depended on the iris pattern quality captured during the iris acquisition. The iris pattern quality can degrade due to the blurry image. Blurry image happened due to the movement during image acquisition and poor camera resolution. Due to that, a deblurring method based on the Wiener filter was proposed to improve the quality of iris pattern. This work is significant since the proposed method can enhance the quality of iris pattern in the blurry image. Based to the results, the proposed method improved the quality of iris pattern in the blurry image. Moreover, it recorded the fastest execution time to improve the quality of iris pattern compared to the other methods.
\end{abstract}

This is an open access article under the CC BY-SA license.

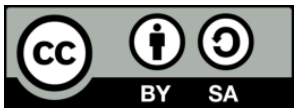

\section{Corresponding Author:}

Shahrizan Jamaludin,

Department of Maritime Technology,

Faculty of Ocean Engineering Technology and Informatics,

Universiti Malaysia Terengganu, Malaysia.

Email: shahrizanj@umt.edu.my

\section{INTRODUCTION}

Security has become the main priority due the current situation and conflict in all parts of the world. In order to improve the security, a lot of money has been spent for the security equipment and personals [1]. The biometric technology has been proposed in order to help the government through their own authorities to identify the terrorists and criminals. This technology is reliable since a lot of biometric data are collected and stored everyday from the immigration department, police department and medical health care [1]. One of the biometric systems is iris recognition, where it can be used for human recognition by using the human irises. It can be implemented on the computer [2], FPGA [3], and GPU [4], with full [1] and partial [5] segmentations. Normally, iris recognition is divided into five main stages which are iris acquisition, iris segmentation, feature extraction, normalization and matching.

The likelihood to locate two persons with a similar iris is estimated at 1 in $10^{72}$ [6]. It is a pigmented tissue and located behind the cornea [7]. Iris is formed in the first trimester of pregnancy and completed in the third trimester. Moreover, the natural test against artifice is provided from its response to the light [8]. Other than that, there is no similar iris between twins due to the complex features such as zig-zag collarette, ridges, rings, freckles, furrows, corona, crypts and arching ligaments [9], which makes the iris recognition is unique for everyone. The advantages of iris recognition are stability over time, easy to use, one unique ID, high recognition accuracy and high security. The iris can be used for as a pass to access the controlled area such as the airport, chemical laboratory, armoury, and many more. 
However, the poor quality of iris images can deteriorate the recognition accuracy of the system. The recognition accuracy is depended on the iris pattern quality captured during the image acquisition. The iris pattern quality can degrade because of the blurry image. Blurry image happened due to the movement during image acquisition and poor camera resolution. Moreover, it can introduce shadow and noise that can degrade the rich texture of iris pattern. Due to that, the Wiener filter is proposed to improve the quality of iris pattern. This work is significant since the proposed method can improve the quality of iris pattern in the blurry image. Due to that, a high-quality iris image and a high-resolution camera are not needed in order to verify the identity of human.

\section{RESEARCH METHOD}

Image deblurring is a process to restore the blurry image. The blurry image happened from the disturbance of scattered light of sun, camera, reflection and lamp. Other than that, the blurry image occurred due to the movement of camera or object during the acquisition process. The inappropriate capturing time can also contribute to the blurry image due to the reduced numbers of photons acquired. Moreover, the blurry image can also be created from the wrong lens angles and poor focus.

Previously, in order to improve the iris image quality, method [10] used the multi-scale retinex and homomorphic filtering to remove shadows and deblur iris image. Then, the histogram analysis was used to enhance the contrast of iris image. Other than that, the spectral curve fitting was used by method [11] for the restoration of defocused iris image. Moreover, the motion blur was improved by the total variation regularized technique [12]. Then in [13], the statistics of local and global iris image were introduced to improve the image. The point spread function (PSF) as in [14] can be implemented to deblurring the image. The Lucy-Richardson algorithm [15] and blind deconvolution algorithm [16] can also be conducted to increase the iris image quality. Other than that, the regularized filter can also be used for deblurring iris image. Image quality assessment [17], hybrid statistical noise suppression [18] and optimum mean [19] can also be used to enhance the quality of image. The latest denoising methods such as [20-24]. However, the deblurring process might require more execution time in order to enhance the iris pattern quality. The deblurring methods stated above required heavy computation, thus more time taken for deblurring process.

In this paper, the Wiener filter is proposed as a deblurring method for iris image. Wiener filter is a deconvolution method implemented when the image frequency and additive noise are known. The Wiener filter can reduce noise with a poor signal-to-noise ratio (SNR). Moreover, the least square restoration technique is used with the Wiener filter. Firstly, the point spread function (PSF) is obtained from the blurry pixel width of the blurry iris image. The PSF is measured to get the spread point of light of the optical system. After that, the camera linear motion and the Gaussian low-pass filter are selected as the constraints of PSF. Finally, the Wiener filter is applied on the iris image. Wiener filter is derived from (1):

$$
W\left(f_{1}, f_{2}\right)=\frac{H^{*}\left(f_{1}, f_{2}\right) S_{x x}\left(f_{1}, f_{2}\right)}{\left|H\left(f_{1}, f_{2}\right)\right|^{2} S_{x x}\left(f_{1}, f_{2}\right)+S_{n n}\left(f_{1}, f_{2}\right)^{\prime}}
$$

where $S_{x x}\left(f_{1}, f_{2}\right)$ is the original iris image power spectra, $S_{n n}\left(f_{1}, f_{2}\right)$ is the noise power spectra and $H\left(f_{1}, f_{2}\right)$ is the blurring filter. The quality of blurry iris image is improved by applying the highpass filter, while the noise is removed with the lowpass filter. Meanwhile, the power spectrum $S_{x x}$ is derived from (2).

$$
S_{x x}=\frac{S_{y y}-S_{n n}}{|H|^{2}}
$$

After that, the proposed method is compared with the Lucy-Richardson algorithm [15] where the Poisson noise is used to enhance the blurry image when the PSF and additive noise information is known. Then, the proposed method is compared with the blind deconvolution algorithm [16] when the PSF and additive noise information is unknown. In this algorithm, PSF is obtained by measuring the width of blurry pixels in the iris image. A few improvements are applied on this algorithm where it can decrease noise effect, eliminate bad pixels and can improvise noise from the camera read-out. 


\section{RESULTS AND DISCUSSION}

The experiment is conducted on the Intel Core i5 $(2.3 \mathrm{GHz})$ processor, $4 \mathrm{~GB}$ RAM memory and MATLAB software. The UBIRIS v1 [25] database is used for this experiment which is collected by SOCIA Lab. The proposed method was implemented on the iris images with two conditions. The first condition was to apply on the entire iris image (full processing). The second condition was the proposed method applied on the iris region only (specific processing). After that, the proposed method was differentiated with the blind deconvolution algorithm and Lucy-Richardson algorithm. Based on Figure 1, the proposed method managed to enhance the iris image quality. The iris images applied with both full and specific processing were improved by using the proposed method. However, the specific processing focused on the iris region only. Meanwhile, the full processing focused on the entire image. Logically, the full processing was wasting time since it required to process the entire image, while the specific processing only on the small part of the image. The iris region should be the only region to be applied with the proposed method since it was full with iris pattern. In the other hand, the other parts of iris image such as sclera and pupil contained meaningless elements for iris recognition. Moreover, the proposed method not only can enhance the iris image quality, but also can add more features into the iris pattern. More features will make that iris more unique and hardly to commit fraud. The recognition accuracy can be improved by the good quality of iris pattern.

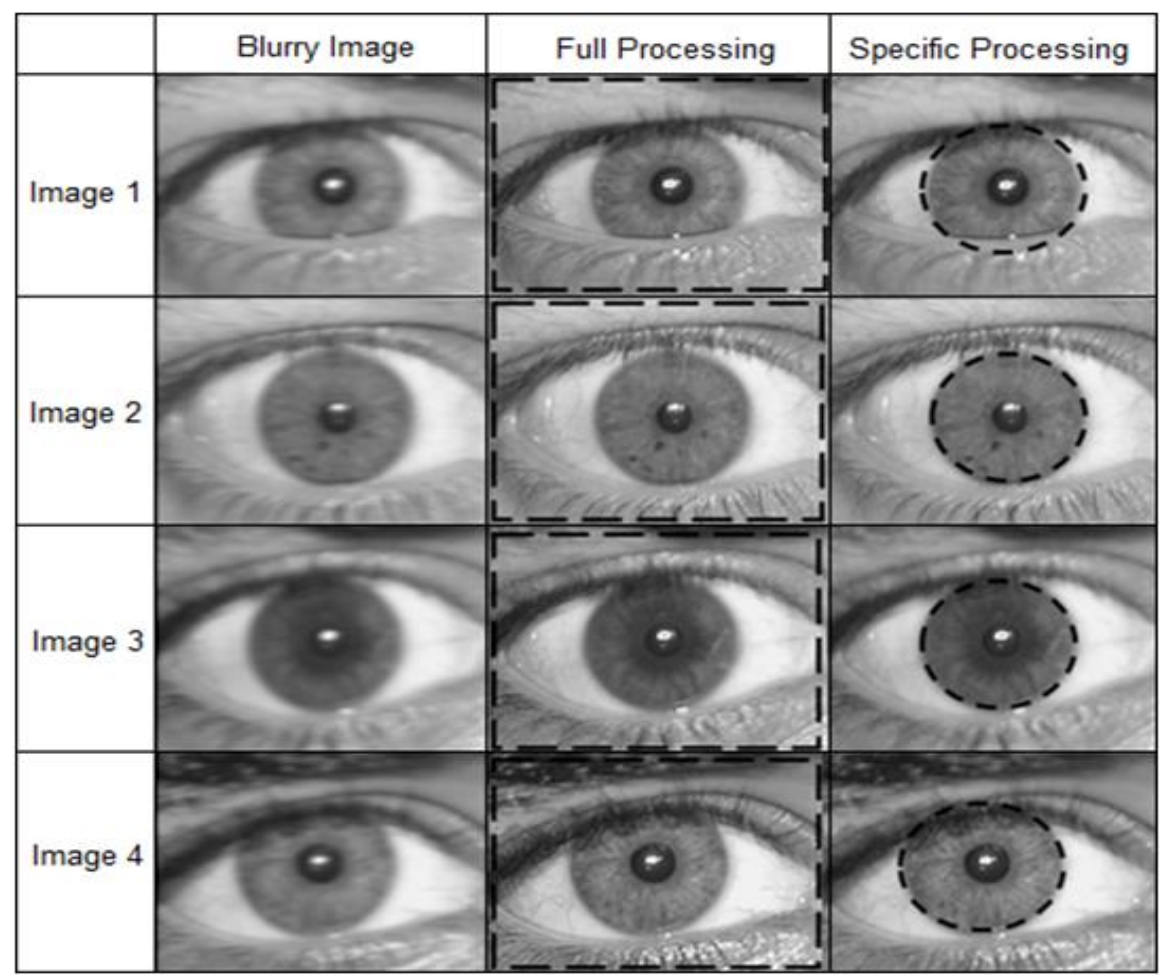

Figure 1. The results of the proposed method

In terms of execution time, the proposed method recorded the fastest execution time as in Table 1. The proposed method was faster and more efficient due to the less complex computation than the blind deconvolution algorithm and Lucy-Richardson algorithm. Moreover, the specific processing of the proposed method recorded a faster execution time than the full processing of the proposed method. For the specific processing, the proposed method was applied on a small region in the image. Meanwhile for the full processing, the proposed method was applied on the entire iris image, thus required more execution time to perform the proposed deblurring process.

Table 1. The results of execution time

\begin{tabular}{lcc}
\hline \multirow{2}{*}{ Methods } & \multicolumn{2}{c}{ Average execution time (s) } \\
\cline { 2 - 3 } & Full processing & Specific processing \\
\hline Blind deconvolution algorithm & 0.807 & 0.783 \\
Lucy-Richardson algorithm & 0.368 & 0.348 \\
Proposed method & 0.067 & 0.063 \\
\hline
\end{tabular}




\section{CONCLUSION}

In this study, a deblurring method based on the Wiener filter was proposed in order to enhance the quality of iris pattern and to reduce the execution time. The proposed method was applied on the iris images with two conditions: on the whole iris image (full processing) and on the specific iris region (specific processing). Based to the results, the proposed method improved the quality of iris pattern in the blurry image. Due to that, a high-quality iris image and a high-resolution camera are not needed to recognize the identity of human. Moreover, the proposed method not only can enhance the quality of iris image, but can also add more features into the iris pattern. Other than that, the proposed method achieved the fastest execution time compared to the others. The proposed method was faster and more efficient due to the less complex computation. For future work, the other deblurring method such as the regularized filter can be implemented on the blurry iris images in order to improve the execution time.

\section{REFERENCES}

[1] S. Jamaludin, N. Zainal, and W. M. Diyana W. Zaki, "The removal of specular reflection in noisy iris image," Journal of Telecommunication, Electronic and Computer Engineering. vol. 8, no. 4, pp. 59-64, 2016.

[2] S. Jamaludin, N. Zainal, and W. M. Diyana W. Zaki, "Iris recognition based on the modified chan-vese active contour," Jurnal Teknologi, vol. 78, no. 10, pp. 13-17, 2016.

[3] T. M. Khan, D. G. Bailey, M. A. U. Khan, and Y. Kong, "Efficient hardware implementation strategy for local normalization of fingerprint images," Journal of Real-Time Image Processing, vol. 16, pp. 1263-1275, 2019.

[4] S. Jamaludin, N. Zainal, and W. M. Diyana W. Zaki, "GPU implementation of sub-iris technique in iris recognition system,” Pertanika Journal of Science and Technology, vol. 25, pp. 263-274, 2017.

[5] S. Jamaludin, N. Zainal, and W. M. Diyana W. Zaki, "Sub-iris technique for non-ideal iris recognition," Arabian Journal for Science and Engineering, vol. 43, no. 12, pp. 7219-7228, 2018.

[6] L. Flom and A. Safir, "Iris recognition system," U.S. Patent no. 4,641,349, Feb. 1987.

[7] U. von Seelen, "Iriscode template compression and its effects on authentication performance," Biometrics Consortium Conference, pp. 1-18, 2004.

[8] J. Daugman, "How iris recognition works," IEEE Transactions on Circuits and Systems for Video Technology, vol. 14, no. 1, pp. 21-30, Jan. 2004.

[9] A. Muron and M. L. Pospisil, "The human iris structure and its usages," Acta Universitatis Palackianae Physica, vol. 39, pp. 87-95, 2000.

[10] D. S. Md Shukri, H. Asmuni, R. M. Othman, and R. Hassan, "An improved multiscale retinex algorithm for motion-blurred iris images to minimize the intra-individual variations," Pattern Recognition Letters, vol. 34, no.9, pp. 1071-1077, 2013.

[11] H. Ren, Y. He, S. Wang, C. Gan, and J. Wang, "Defocused iris image restoration based on spectral curve fitting," Chinese Conference on Biometric Recognition, pp. 338-344, 2013.

[12] F. Alaoui, K. Assid, V. Dembele, and A. Nassim, "Application of blind deblurring algorithm for iris biometric," International Journal of Computer Applications, vol. 79, no. 3, pp. 11-15, 2013.

[13] L. Ren, X. Huang, and R. Yang, "Iris deblurring method based on global and local iris image statistics," U.S. Patent no. $8,374,389,2013$.

[14] J. Liu, Z. Sun, and T. Tan, "Iris image deblurring based on refinement of point spread function," Chinese Conference on Biometric Recognition, pp. 184-192, 2012.

[15] P. Anjaiah, "An approach to enhancing security of biometric image with retrieval system," Anveshana's International Journal of Research in Engineering and Applied Sciences, vol. 2, no. 3, pp. 147-154, 2017.

[16] F. L. de A. Junior, R. N. Franca, and D. Z. Rodriguez, "Image quality treatment to improve iris biometric systems," INFOCOMP Journal of Computer Science, vol. 16, no. 1-2, pp. 21-30, 2017.

[17] F. Mokhtar, R. Ngadiran, T. Basheer, and A. N. A. Rahim, "Analysis of wavelet-based full reference image quality assessment algorithm," Bulletin of Electrical Engineering and Informatics, vol. 8, no. 2, pp. 527-532, 2019.

[18] S. Rajkumar and G. Malathi, "An efficient image denoising approach for the recovery of impulse noise," Bulletin of Electrical Engineering and Informatics, vol. 6, no. 3, pp. 281-286, 2017.

[19] W. A. Mustafa, M. M. M. A. Kader, and Z. I. A. Khalib, "Improved wolf algorithm on document images detection using optimum mean technique," Bulletin of Electrical Engineering and Informatics, vol. 8, no. 2, pp. 551-557, 2019.

[20] L. Abderrahim, M. Salama, and D. Abdelbaki, "Novel design of a fractional wavelet and its application to image denoising," Bulletin of Electrical Engineering and Informatics, vol. 9, no. 1, pp. 129-140, 2019.

[21] C. Wu, X. Ma, and W. Wang, "Hyperspectral image denoise based on curvelet transform combined with weight coefficient method," Journal of Intelligent and Fuzzy Systems, vol. 37, no. 4, pp. 4425-4429, 2019.

[22] P. Sahu, "A novel approach to denoise 2d-images from different noise signals using combination of various filters," International Journal of Scientific Research in Science, Engineering and Technology, vol. 2, no. 3, pp. 728-732, 2016.

[23] P. Jain, A. Mathur, and H. Dhabhai, "Analysis of MRI image de-noising technique," International Journal of Computer Science and Mobile Computing, vol. 8, no. 5, pp. 171-175, 2019.

[24] R. M. Farouk, M. Elsayed, and M. Aly, "Medical image denoising based on log-gabor wavelet dictionary and k-svd algorithm," International Journal of Computer Applications, vol. 141, no. 1, pp. 27-32, 2016.

[25] H. Proenca and L. A. Alexandre, "UBIRIS: A noisy iris image database," International Conference on Image Analysis and Processing, pp. 970-977, 2005. 